International Journal of Rotating Machinery

1999, Vol. 5, No. 4, pp. 295-304

Reprints available directly from the publisher

Photocopying permitted by license only
(C) 1999 OPA (Overseas Publishers Association) N.V

Published by license unde the Gordon and Breach Science Publishers imprin Printed in Malaysia.

\title{
The Dynamics of Rotor with Rubbing*
}

\author{
JERZY T. SAWICKI ${ }^{\mathrm{a}, \dagger}$, JOE PADOVAN ${ }^{\mathrm{b}}$ and RABIH AL-KHATIB ${ }^{\mathrm{a}}$ \\ a Department of Mechanical Engineering, Cleveland State University, Cleveland, OH 44115-2425, USA, \\ ${ }^{\mathrm{b}}$ Department of Mechanical Engineering, University of Akron, Akron, OH 44325-3903, USA
}

(Received 24 April 1998; In final form 7 July 1998)

\begin{abstract}
This paper presents the description of some phenomena associated with dynamic behavior of rotors interacting with stationary components. Numerical simulations show rotor vibration spectrum rich in subharmonic, quasi-periodic, and chaotic vibrations. The nonlinear calculation techniques are applied to demonstrate the changes of the vibration patterns for different operating conditions. Some conclusions are discussed with regard to unique characteristics of rub-induced rotor response, initial conditions, as well as appropriate ranges of system parameters. Of special interest are the changes in the apparent nonlinearity of the system dynamics as rubs are induced at different rotor speeds. In particular, starting with 2 nd order sub/superharmonics, which are symptomatic of quadratic nonlinearity, progressively higher order polynomial behavior is excited, i.e., cubic, giving rise to 3rd order sub/ superharmonics. As the speed is transitioned between such apparent nonlinearities, chaotic like behavior is induced because of the lack of whole or rational tone tuning between the apparent system frequency and the external source noise. The cause of such behavior will be discussed in detail along with the results of several parametric studies.
\end{abstract}

Keywords: Rotor, Vibration, Chaos, Poincaré map, Bifurcation diagram, Rub impact

\section{INTRODUCTION}

It is widely recognized that under certain conditions rotating machinery exhibit vibrations which have chaotic content, i.e., present unpredictable behavior. Such behavior is driven by the existence of nonlinearities in the system which could have many roots, e.g., interaction of the rotating and stationary components (Ehrich, 1966; 1992; Bently,
1974; Childs, 1982; Muszynska, 1984; Beatty, 1985; Padovan and Choy, 1987; Geradin and Kill, 1988; Kraker et al., 1988; Choy et al., 1988; Goldman and Muszynska, 1993; 1994; Adams and AbuMahfouz, 1994). Many papers have employed bifurcation diagrams and/or Poincaré maps to report on the chaotic nature of rub interactions, for example see Ehrich (1966, 1992), Goldman and Muszynska (1993, 1994), Adams and Abu-Mahfouz

* This paper was originally presented at ISROMAC-7.

${ }^{\dagger}$ Corresponding author. Tel.: 2166872565 . Fax: 2166879280. 
(1994). None though have explained the occurrence of such phenomena in terms of induced nonlinearities.

In this context, based on a three mass rotor system, this paper will track changes in the apparent system nonlinearity induced by rub dynamics as the speed of the rotor system is progressively increased. By employing an FFT analysis to monitor the frequency content of the system rotor output, the existence of different forms of harmonics and sub/ super harmonics is utilized to define the predominating form of system nonlinearity, i.e., softening vs. hardening polynomial vs. transcendental. The occurrence of such behavior will be employed to explain the existence of chaotic like response, wherein due to the lack of whole tone tuning between the apparent system frequency and the driving input, the rotor continuously precesses into a sequence of apparently nonrecurring rub sites. Finally, the form of nonlinearity will also be utilized to explain the apparent domain of sub vs. super harmonics as damping is progressively increased.

\section{MULTI-MASS RUB-IMPACT ROTOR MODEL}

In previous works considering rub impact induced chaos of rotor dynamics, prototypically single mass rotor systems were considered. Here a multi-mass system is treated so as to assess the degree to which apparent chaos is distributed throughout the rotor system. To further complicate the dynamics an axially asymmetric mass distribution with anisotropic gravity effects is considered. This enables some mass stations to rub while others are impact free. As a further feature, the operating conditions are ranged from intermittent rubs at lower speeds to a full rubs at high speeds. The salient features of the simulation are given in the following subsections.

\section{Modeling of the Multi-mass Rotor}

The equations of motion for multi-mass rotor model, limited to lateral vibrations and no gyro- scopic effects, can be written as follows:

$$
M \ddot{q}+D \dot{q}+K q=f_{\mathrm{e}}+f_{\mathrm{r}}+G,
$$

where $q^{\mathrm{T}}=\left[\mathbf{x}^{\mathrm{T}} \mathbf{y}^{\mathrm{T}}\right]$ is the displacement vector, $f_{\mathrm{e}}, f_{\mathrm{r}}$, and $G$ are the unbalance excitation force, nonlinear rubbing interaction force, and constant gravity force vectors, respectively.

Let $\Phi$ be the modal matrix $(n \times n)$, where $n$ is the number of modes,

$$
\Phi=\left[\phi_{1}, \phi_{2}, \ldots, \phi_{n}\right],
$$

and $\phi_{i}=\left[\phi_{i 1}, \phi_{i 2}, \ldots, \phi_{i n}\right]^{\mathrm{T}}$ is the $i$ th mode of the system. Then the equation of motion (Eq. (1)) can be represented in a modal form as

$$
M_{m} \ddot{q}_{m}+D_{m} \dot{q}_{m}+M_{m} \Omega^{2} q_{m}=\Phi^{\mathrm{T}}\left(f_{\mathrm{e}}+f_{\mathrm{r}}+G\right),
$$

where

$$
\begin{gathered}
\Phi^{\mathrm{T}} M \Phi=M_{m}, \\
\Phi^{\mathrm{T}} K \Phi=M_{m} \Omega^{2}=\operatorname{diag}\left(\omega_{i}^{2}\right), \\
D_{m}=\Phi^{\mathrm{T}} D \Phi,
\end{gathered}
$$

and $\omega_{i}$ is the $i$ th natural frequency. The modal mass $\left(M_{m}\right)$ and stiffness $\left(K_{m}\right)$ matrices are diagonal, however the modal damping matrix $\left(D_{m}\right)$ could be fully populated. It is shown elsewhere (Gawronski and Sawicki, 1997) that for small damping $D_{m}$ can be replaced with its diagonal part and the system dynamics changes are negligible. Thus, in further considerations the damping matrix $D_{m}$ is assumed to be a diagonal one.

Left multiplication of Eq. (3) by $M_{m}^{-1}$ yields

$$
\ddot{q}_{m}+M_{m}^{-1} D_{m} \dot{q}_{m}+\Omega^{2} q_{m}=M_{m}^{-1} \Phi^{\mathrm{T}}\left(f_{\mathrm{e}}+f_{\mathrm{r}}+G\right),
$$

and conventionally, one can normalize damping as follows:

$$
M_{m}^{-1} D_{m}=2 Z \Omega, \quad Z=\operatorname{diag}\left(\xi_{1}, \xi_{2}, \ldots, \xi_{n}\right),
$$

where $\xi_{i}$ are the modal damping coefficients, and for subcritical damping $\xi_{i}<1, i=1, \ldots, n$. 
Therefore, it can be assumed that the multi-mode modal model (Eq. (5)) of the rotor consists of $n$ concentrated masses supported on elastic massless shaft.

\section{Rub-impact Model}

The kinematics of the rub-impact model is shown in Fig. 1. It is postulated that in the case of contact of the $\ell$ th disk with the housing, the following condition is satisfied:

$$
\delta_{\ell}=\frac{\delta_{\ell}^{*}}{C}-1=\frac{\sqrt{\left(x_{\ell}^{2}+y_{\ell}^{2}\right)}}{C}-1 \geq 0,
$$

where $\delta_{\ell}^{*}$ is the relative radial displacement of the $\ell$ th disk with respect to the housing, $x_{\ell}, y_{\ell}$ are lateral displacements (components of physical coordinate $q_{\ell}$ corresponding to the $\ell$ th disk), and $C$ is the concentric radial clearance between any disk (assumed the same for all disks) and housing/stator.

In addition, it is assumed that as a result of rubbing, the rotor stiffness increases and induced rubbing force $f_{r \ell}$ is the resultant of nonlinear

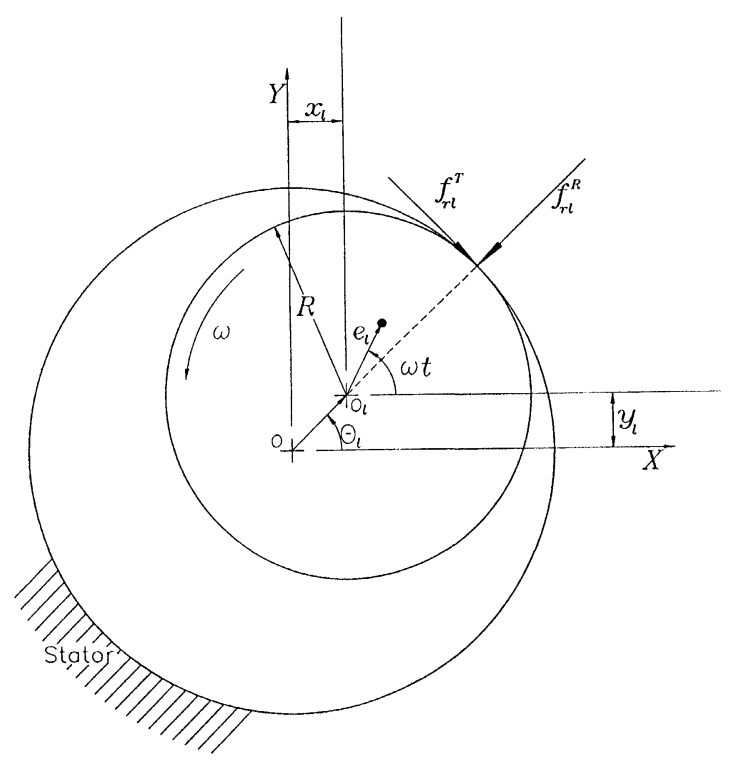

FIGURE 1 Rub-impact model. contact radial load, $f_{r \ell}^{\mathrm{R}}$, and nonlinear tangential friction load, $f_{r \ell}^{\mathrm{T}}$, which are related as

$$
\left\|f_{r \ell}^{\mathrm{T}}\right\|=\mu\left\|f_{r \ell}^{\mathrm{R}}\right\|,
$$

where $\mu$ is the Coulomb friction coefficient. The direction of the frictional force (essential for transient analysis) depends on sign of the expression for the linear velocity of the point of contact, i.e.,

$$
V_{\ell}=R_{\ell} \omega+\Omega_{\ell} \delta_{\ell}^{*}
$$

where $\omega$ is the shaft angular speed, and $\Omega_{\ell}$ is the whirling angular speed of the $\ell$ th disk calculated as follows:

$$
\Omega_{\ell}=\frac{x_{\ell} \dot{y}_{\ell}+\dot{x}_{\ell} y_{\ell}}{\left(\delta_{\ell}^{*}\right)^{2}}
$$

If the contact condition (Eq. (7)) is satisfied, then it is assumed that the induced rubbing force depends exponentially on the orbital radius of the shaft, thus for $\ell$ th disk it takes the following form:

$$
f_{r \ell}=\left\{\begin{array}{l}
f_{r \ell x} \\
f_{r \ell y}
\end{array}\right\}=\left\{\begin{array}{l}
k_{\mathrm{H}} \delta_{\ell}^{\alpha}\left(-\cos \theta_{\ell}+\mu \sin \theta_{\ell}\right) \\
k_{\mathrm{H}} \delta_{\ell}^{\alpha}\left(-\sin \theta_{\ell}-\mu \cos \theta_{\ell}\right)
\end{array}\right\} .
$$

\section{SOLUTION STRATEGY}

The equations of motion (Eq. (1)) were numerically integrated using the predictor-corrector EulerNewmark technique with the time integration step of $2 \pi /(720 \omega)$. Bifurcation diagrams, Poincaré maps and power spectra are used for presentation and analysis of the nonlinear system dynamical behavior.

\section{Bifurcation Diagram}

The dynamics may be viewed globally over a range of parameter values, thereby allowing simultaneous comparison of periodic and chaotic forms of behavior. The bifurcation diagram provides a 
summary of the essential dynamics and is therefore a useful method for acquiring such overview. To compute a bifurcation diagram, the differential equations of motion (Eq. (5)) were integrated over a wide range of the rotor speed. The latter was varied at a constant step (i.e. $2 \mathrm{rad} / \mathrm{s}$ ) and the state variables at the end of each integration were used as the initial conditions for the solution at the next speed. At every rotative speed, the first 200 cycles were discarded to exclude the transient state of motion, and the steady state positions for the next 50 cycles were recorded.

\section{Poincaré Map}

A Poincaré mapping is closely related to the stroboscopic portraits of motion at the specific times, i.e., $t=m T$, with $T$ being the period of excitation and $m=1,2, \ldots$ It simplifies the geometric description of the dynamics by removing one of the state space dimensions, and contains information about the periodicity, quasi-periodicity, chaoticity, and bifurcations of the system's dynamics.

\section{FFT Spectrum}

The FFT map/spectrum was calculated to show the spectral components of the motion. Note the window of the FFT is taken to be the last steady state 50 system rotations. This condition was reached by allowing the inherent system hysteresis damp out all starting effects in the first 200 revolutions. If the motion is periodic or quasiperiodic, then the power spectrum will consist of a sequence of spikes at the fundamental frequencies, their harmonics, and the frequencies that are the sums and differences of the various frequencies. For the nonperiodic motion (e.g., chaotic), the power spectrum shows a random broadband character.

\section{RESULTS AND DISCUSSION}

Before overviewing the results of the numerical parametric studies, it is worthwhile to review various properties of the vibration response of nonlinear systems. Recalling the comments of Stoker (1950), a literal multitude of harmonics can be excited by nonlinearity. Generally, nonlinearity can be defined either by polynomials or transcendental functions. Noting Eq. (5)

$$
\begin{aligned}
\ddot{q}_{m} & +M_{m}^{-1} D_{m} \dot{q}_{m}+\Omega^{2} q_{m}+F_{N}\left(q_{m}\right) \\
& =M_{m}^{-1} \Phi^{\mathrm{T}}\left(f_{\mathrm{e}}+G\right),
\end{aligned}
$$

where $F_{N}\left(q_{m}\right)=-M_{m}^{-1} \Phi^{\mathrm{T}} f_{\mathrm{r}}$ is the nonlinear restoring function which can take the following forms:

(a) single-term polynomial (integer)

$$
F_{N}\left(q_{m}\right)=\mu_{m}\left(q_{m}\right)^{n}, \quad n \in[2,3,4, \ldots),
$$

(b) multi-term polynomial (integer)

$$
F_{N}\left(q_{m}\right)=\sum_{n=2} \mu_{m n}\left(q_{m}\right)^{n}
$$

(c) single-term irrational polynomial

$$
F_{N}\left(q_{m}\right)=\mu_{m}\left(q_{m}\right)^{\xi} \quad(\xi-\text { irrational number })
$$

(d) multi-term irrational polynomial

$$
\begin{gathered}
F_{N}\left(q_{m}\right)=\sum_{\substack{n=1 \\
\text { irrational numbers })}} \mu_{m n}\left(q_{m}\right)^{\xi_{n}} \quad\left(\xi_{n}, n \in[1,2, \ldots)\right. \\
\end{gathered}
$$

(e) transcendental function

$$
F_{N}\left(q_{m}\right)=\gamma\left(q_{m}\right)
$$

where here $\gamma$ is a transcendental function. Depending on the choice of the irrational number families appearing in Eqs. (14) and (15), a whole range of functional types can be represented.

In the context of the frequency, depending on the driving input, i.e., rotating unbalance force, which is periodic/harmonic, a complex mix of function types can evolve in the output spectrum. 
For instance, for a quadratic integer polynomial,

$$
F_{N}\left(q_{m}\right) \propto\left(q_{m}\right)^{2} .
$$

Generally a harmonic excitation $\cos (\Omega t)$ will lead to a solution of the form (Padovan and Zeid, 1981; Padovan, 1986)

$$
\sum_{n} \alpha_{n}(t) \cos (2 n \Omega t)+\sum_{n} \beta_{n}(t) \cos \left(\frac{1}{2 n} \Omega t\right)
$$

which consists of harmonic and sub/super harmonic components. As was demonstrated by Padovan and Zeid (1981) and Padovan (1986), the subharmonics are a natural outgrowth of the steady state system behavior while superharmonics grow out of the system transient properties. Specifically, superharmonics have a tendency to decay with time due to system hysteresis. For rub impact problems, the succession of rotor casing hits tends to sustain the transient behavior, thereby exciting the superharmonics. In the case of a cubic integer polynomial

$$
F_{N}\left(q_{m}\right) \propto\left(q_{m}\right)^{3},
$$

a harmonic input $\cos (\Omega t)$ will lead to an output of the form

$$
\sum_{n} \alpha_{n}(t) \cos (3 n \Omega t)+\sum_{n} \beta_{n}(t) \cos \left(\frac{1}{3 n} \Omega t\right)
$$

which consists of 3rd order sub and superharmonics. For integer polynomials, whether single or multiple term, the exact nature of the type of harmonic/sub/super harmonic can easily be worked out (Padovan and Zeid, 1981; Padovan, 1986).

Generally, for harmonic inputs, such nonlinearities tend to give rise to complex but more or less nonchaotic responses. This is an outgrowth of the exact tuning occurring because of the integer nature of the cosine/sine families of functions. In the case of irrational functions, generally there is no exact integer tuning between the input/output, thus leading to precessing orbits. The precessing becomes stronger/more chaotic the more the $\xi, \xi_{n}$ families of powers diverge from integer numbers.
As a final feature of nonlinearity, we must discuss the issue of the oddness/evenness/asymmetry of $F_{N}\left(q_{m}\right)$. Typically $F_{N}$ is treated as being an odd function - one in which the restoring forces are the same magnitude in compression and tension. If one were to investigate large deformation mechanics which involves a 2nd Piola-Kirchhoff/GreenLagrange stress/strain measure for a nonlinear medium, then a more or less asymmetric type of nonlinearity occurs (Padovan, 1986). For linear Hookean materials in the elastic limit, the dominating nonlinearity is a mix of quadratic and cubic terms. Since the quadratic terms are even functions, they lead to asymmetry, i.e., hardening in one direction and softening in the other. The cubic terms are odd functions, they yield a symmetrical behavior. When combined, the overall behavior is asymmetric.

Averaging the rotor response behavior over several revolutions, the free and impacted behavior tends to reflect a global apparent system nonlinearity. With the introduction of directional effects such as gravity, static loading, or variable clearance configurations, a degree of asymmetry is induced in the orientation undergoing the most rub-bounce events. This implies that $F_{N}$ is dominated by even order polynomials. As we saw earlier, if the spectrum exhibits multiples of $1 / 2 n$ or $2 n$ in the frequencies of excitation then $(q)^{2}$-type nonlinearity predominates the response. If on the other hand $1 / 3 n$ or $3 n$ spectra are excited, then $(q)^{3}$-type behavior is dominant.

As a rule of thumb, spectra formed from the even ordered multiple $(1 / 2 n$ or $2 n)$ of $\omega$ are a result of system asymmetry, while the multiples $1 /(2 n+1)$, $2 n+1$ are typically due to symmetrical type behavior. All appears that $\left(\frac{1}{2}, 2\right)$ multiples predominate the asymmetric behavior and $\left(\frac{1}{3}, 3\right)$ the symmetrical.

For a system transitioning between such effects, the apparent nonlinearity is essentially fractional in nature, wherein no rational multiples of the driving frequency occur. Such behavior is perhaps the most prevalent mode of the response. Under such conditions, the impact sites will precess around the 
casing leading to apparent chaos. When the behavior is near an odd and even whole integer multiple of $\omega$, then the precessing is very slow leading to apparently overlapping trajectory bounds.

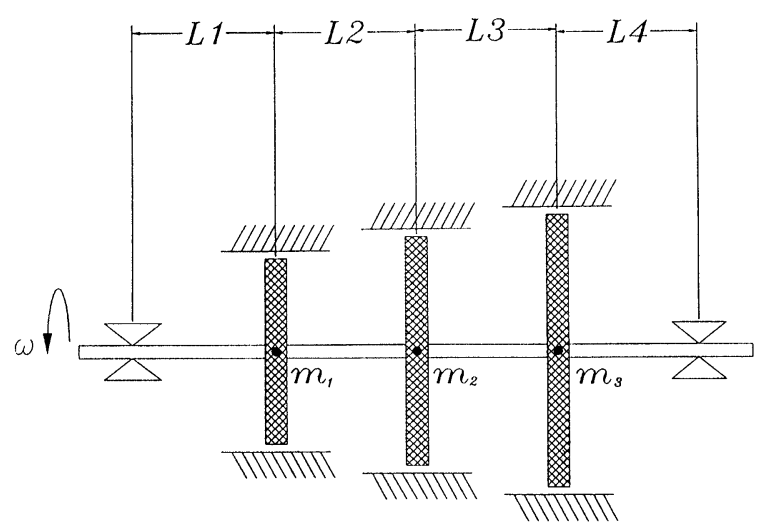

FIGURE 2 Three-disc rotor model.
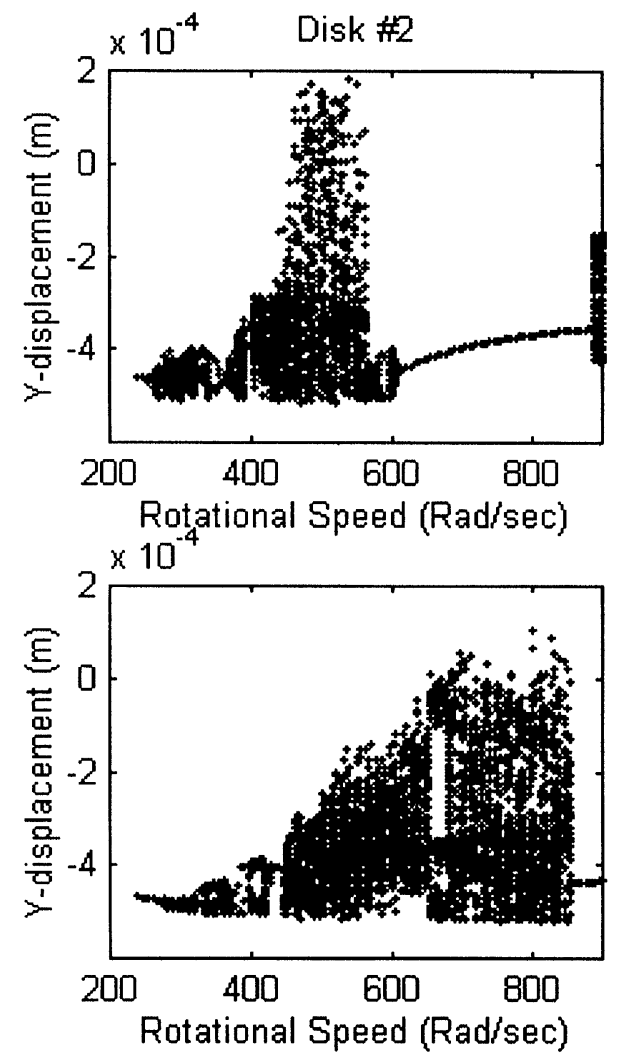

To illustrate various of the effects, the axially asymmetric rotor system described in Figs. 1 and 2 was excited by an unbalance attached to disc 2 . The unbalance eccentricity was assumed to be $e=5 \times 10^{-4} \mathrm{~m}$. The other system's parameters are: $l_{1}=0.25, \quad l_{2}=l_{4}=0.26, \quad l_{3}=0.27 \mathrm{~m}, \quad$ and $m_{1}=2, m_{2}=4$ and $m_{3}=6 \mathrm{~kg}$. The system's natural frequencies are: 110,438 and $931 \mathrm{rad} / \mathrm{s}$. Figures 3 and 4 illustrate bifurcation diagrams showing the response for different damping levels and interfacial rub friction. The results in Fig. 3 clearly suggest the possibility of the use of chaos mapping of monitored vibration signals as a diagnostic tool to detect some rotating machinery malfunctions. Orbits and Poincaré maps are shown in Fig. 5(a) and (b).

As the speed of rotation is ranged over the interval illustrated in Fig. 4, a wide variety of
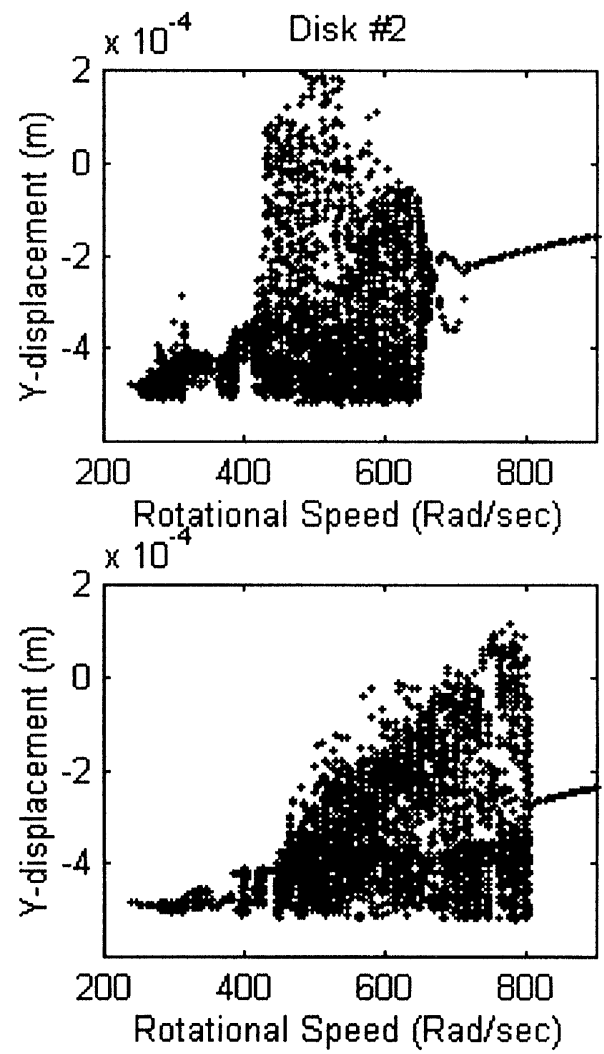

FIGURE 3 Bifurcation diagrams showing response for different damping (first row figures: $\xi=0.2$, second row $\xi=0.3$ ) and friction coefficients (left column figures: $\mu=0.15$, right column $\mu=0.03$ ) with rotational speed as the control parameter. 

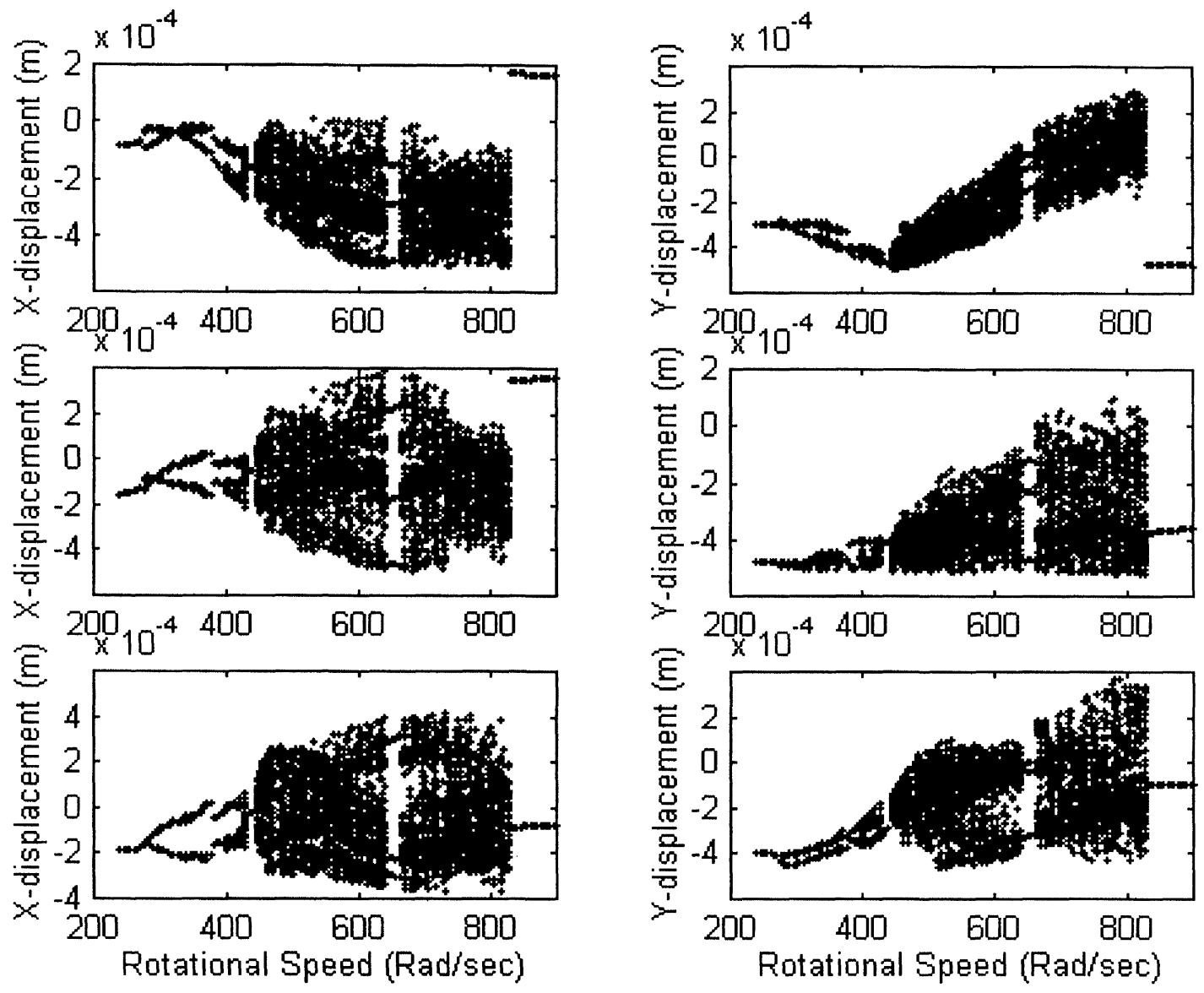

FIGURE 4 Bifurcation diagrams showing lateral $x$ and $y$ responses for each disk (first row: disk \#1, last row: disk \#3).

response characteristics are excited. These are best illustrated by the power spectrum. Figure 6 illustrates the evolving influence of the apparent system nonlinearity. For low velocities, $(1 / 2 n, 2 n)$ type harmonics predominate. These are the result of a quadratic type apparent spring stiffness. As the speed is increased, the $(1 / 2 n, 2 n)$ behavior is centered in growing bands as the nonlinearity picks up irrational powers or becomes transcendental functionally, wherein parts of the solution are detuned from the harmonic input leading to a chaotic like response. Note, for low rotor speeds, the rubbing is predominantly centered at the bottom clearance circle. This is a direct result of the strong gravity effects, which are the primary reason for the directional stiffness variation leading to the quadraticlike behavior. As the speed is further increased, the rubbing spreads from the bottom leading to weaker directionality in the apparent stiffness resulting in a more symmetrical apparent stiffness. This is a direct result of the increased momentum overcoming gravitational effects for the given rotor. Note, as this stiffness becomes more symmetrical, the bilinear stiffening caused by the rubbing impact tends to be cubic in nature giving rise to peaks at $(1 / 3 n, 3 n)$. At the early stages such peaks are centered in bands due to the existence of irrational/ transcendental functionality, i.e., detuning behavior. As the rubbing zone spreads the $\left(\frac{1}{3}, 3\right)$ peaks become crisper. 


\section{SUMMARY AND CONCLUSIONS}

This paper presented the analytical and numerical simulation of the unbalanced multi-disk rotor with rubbing. By employing nonlinear vibration theory, many of the phenomena evolving out of rub impact in rotating systems have been addressed. This included such issues as:

(1) the form of sub/super harmonics and their varying causes;
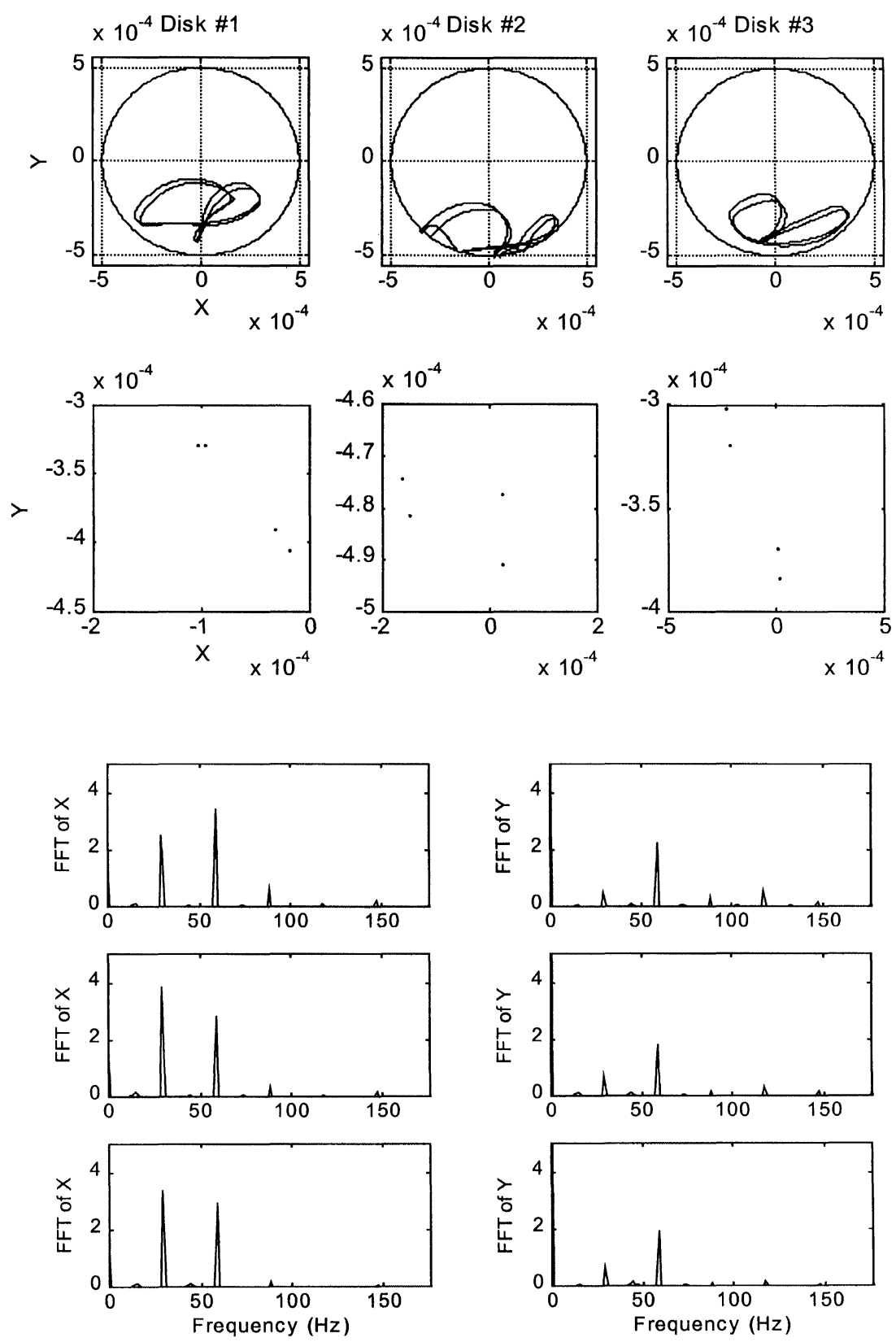

FIGURE 5(a) Orbits, Poincaré maps and power spectrum for rotational speed $\omega=369 \mathrm{rad} / \mathrm{s}$. 

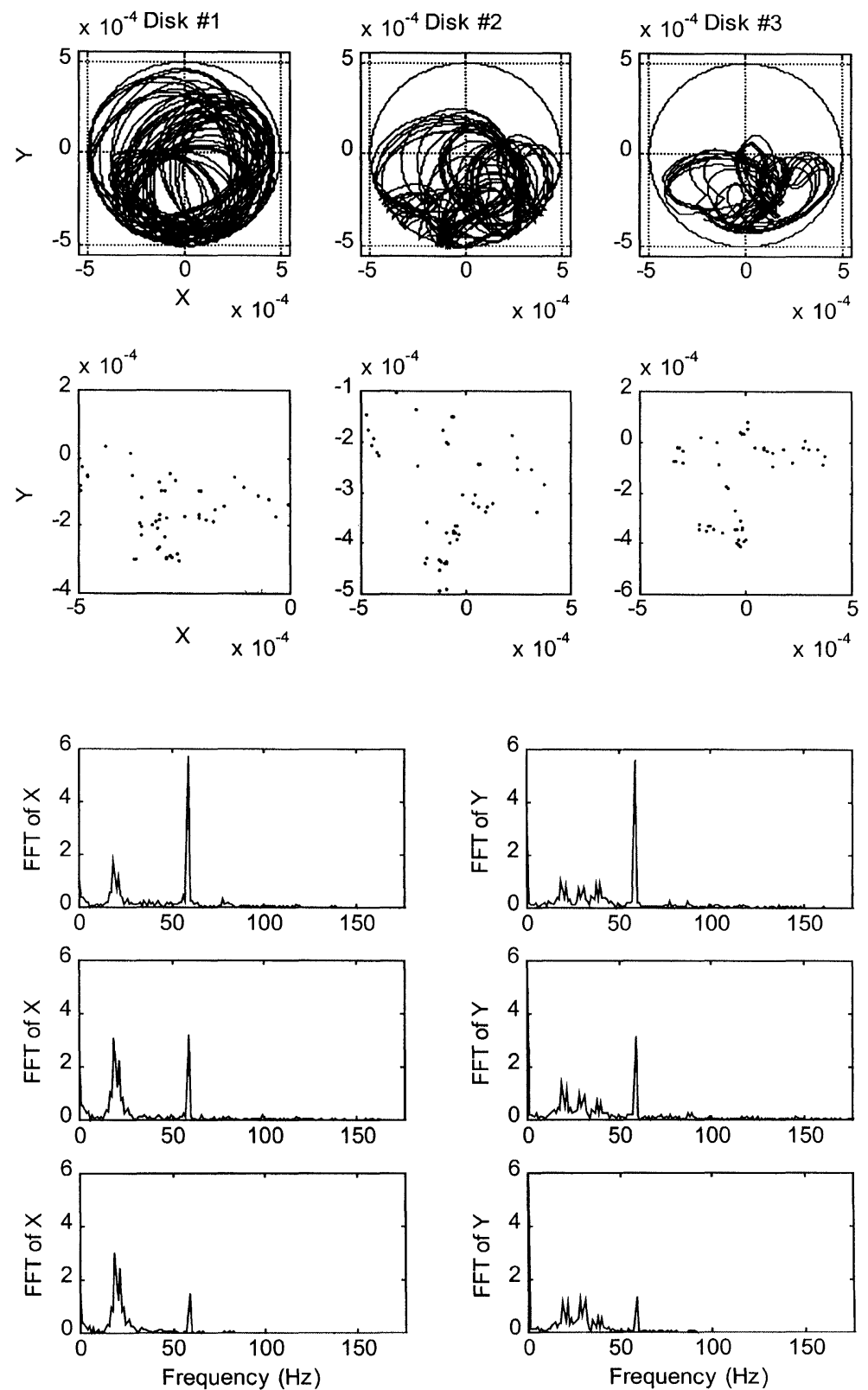

FIGURE 5(b) Orbits, Poincaré maps and power spectrum for rotation speed $\omega=625 \mathrm{rad} / \mathrm{s}$.

(2) the effects of asymmetry on the loading field;

(3) the influence of rotor speed in changes from asymmetry to apparent symmetry;

(4) the relationship between the types of harmonics and the evolving nonlinearity induced by intermittent rub bounces;
(5) the numerical study of the bifurcation of the parameter dependent system; and

(6) the time evolution description of the system nonlinear behavior via Poincaré map technique. 

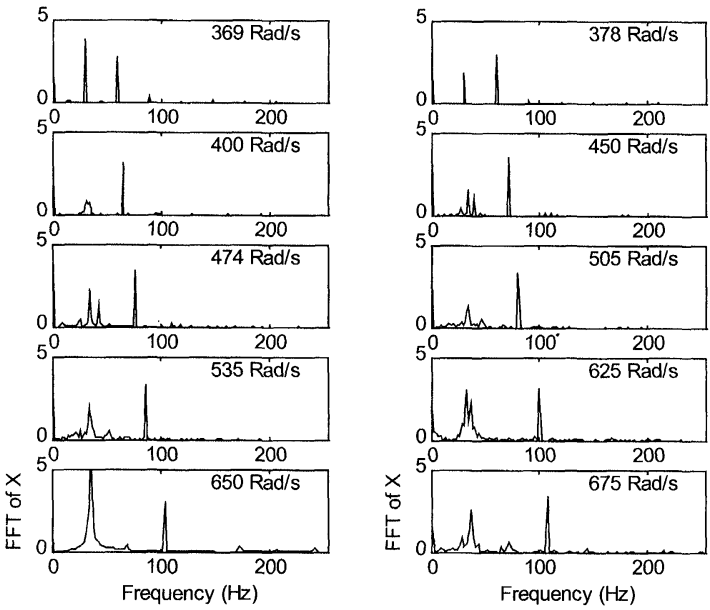

FIGURE 6 FFT spectra for $x$-displacement of disk \#2 at different rotor speeds.

\section{Acknowledgment}

This work was carried out as part of a research project supported by Cleveland State University Research Council's Established, Full-time Faculty Research Development (EFFRD) Program, 1996.

\section{References}

Adams, M.L. and Abu-Mahfouz, I.A. (1994) Exploratory research on chaos concepts as diagnostic tools for assessing rotating machinery vibration signatures, Proc. of The Fourth Int. Conference on Rotor Dynamics, Chicago, pp. 29-39.

Beatty, R.F. (1985) Differentiating rotor response due to radial rubbing, ASME Journal of Vibration, Acoustics, Stress and Reliability in Design, 107, 151-160.
Bently, D.E. (1974) Forced subrotative speed dynamic action of rotating machinery, ASME Publication, 74-Pet-16, Petroleum Mechanical Engineering Conference, Dallas, Texas.

Childs, D.W. (1982) Fractional-frequency rotor motion due to nonsymmetric clearance effects, ASME Journal of Energy and Power, 104, 533-541.

Choy, F.K., Padovan, J. and Li, W.H. (1988) Rub in high performance turbomachinery, modeling, solution methodology and signature analysis, Mechanical Systems and Signal Processing, 2(2), 113-133.

Ehrich, E.F. (1966) Subharmonic vibrations of rotors in bearing clearance, ASME Paper 66-MS-1.

Ehrich, F. (1992) Observations of subcritical superharmonic and chaotic response in rotordynamics, ASME Journal of Vibration and Acoustics, 114, 93-100.

Gawronski, W. and Sawicki, J.T. (1997) Response errors of nonproportionally lightly damped structures, Journal of Sound and Vibration, 200, 543-550.

Geradin, M. and Kill, N. (1988) Non-linear dynamic analysis of flexible rotors, Proc. IMechE, C283/88, pp. 627--634.

Goldman, P. and Muszynska, A. (1994) Dynamic effects in mechanical structures with gaps and impacting: order and chaos, ASME Journal of Vibration and Acoustics, 116, $541-547$.

Goldman, P. and Muszynska, A. (1993) Chaotic behavior of rotor/stator systems with rubs, ASME Turbo Expo Conference, Cincinnati.

Kraker, D., Crooijmans, M.T.M. and Campen, D.H. (1988) The dynamics of a rotor with rubbing, Proc. IMechE, C284/88, pp. $297-303$.

Muszynska, A. (1984) Partial lateral rotor to stator rubs, Proc. IMechE, C281/84, pp. 327-335.

Padovan, J. (1986) Nonlinear spectral characteristics of large deformation elasticity theory, International Journal of Engineering Science, 24, 1517-- 1535.

Padovan, J. and Choy, F.K. (1987) Nonlinear dynamics of rotor/ blade/casing rub interactions, ASME Journal of Turbomachinery, 108, 527-534.

Padovan, J. and Zeid, I. (1981) Sub/superharmonic oscillations and perturbation procedure, International Journal of Nonlinear Mechanics, 16, 465-478.

Stoker, J.J. (1950) Nonlinear Vibrations in Mechanical and Electrical Systems, Wiley-Interscience, New York. 


\section{ait \\ ENERGY MATERIALS}

M A N E Y publishing

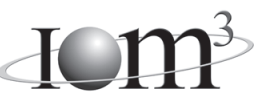

\section{Materials Science \& Engineering for Energy Systems}

Maney Publishing on behalf of the Institute of Materials, Minerals and Mining

The Institute of Materials, Minerals \& Mining

Economic and environmental factors are creating ever greater pressures for the efficient generation, transmission and use of energy. Materials developments are crucial to progress in all these areas: to innovation in design; to extending lifetime and maintenance intervals; and to successful operation in more demanding environments. Drawing together the broad community with interests in these areas, Energy Materials addresses materials needs in future energy generation, transmission, utilisation, conservation and storage. The journal covers thermal generation and gas turbines; renewable power (wind, wave, tidal, hydro, solar and geothermal); fuel cells (low and high temperature); materials issues relevant to biomass and biotechnology; nuclear power generation (fission and fusion); hydrogen generation and storage in the context of the 'hydrogen economy'; and the transmission and storage of the energy produced.

As well as publishing high-quality peer-reviewed research, Energy Materials promotes discussion of issues common to all sectors, through commissioned reviews and commentaries. The journal includes coverage of energy economics and policy, and broader social issues, since the political and legislative context influence research and investment decisions.

\section{CALL FOR PAPERS}

Contributions to the journal should be submitted online at http://ema.edmgr.com

To view the Notes for Contributors please visit: www.maney.co.uk/journals/notes/ema

Upon publication in 2006, this journal will be available via the Ingenta Connect journals service. To view free sample content online visit: www.ingentaconnect.com/content/maney

For further information please contact:

Maney Publishing UK

Tel: +44 (0)113 2497481 Fax: +44 (0)1132486983 Email: subscriptions@maney.co.uk

or

Maney Publishing North America

Tel (toll free): 8662975154 Fax: 6173546875 Email: maney@maneyusa.com

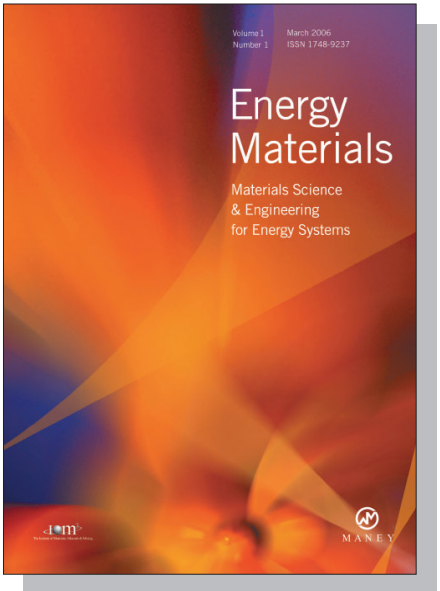

EDITORS

Dr Fujio Abe

NIMS, Japan

Dr John Hald, IPL-MPT, Technical University of Denmark, Denmark

Dr R Viswanathan, EPRI, USA

\section{SUBSCRIPTION INFORMATION}

Volume 1 (2006), 4 issues per year

Print ISSN: 1748-9237 Online ISSN: 1748-9245

Individual rate: $£ 76.00 / U S \$ 141.00$

Institutional rate: $£ 235.00 /$ US $\$ 435.00$

Online-only institutional rate: $£ 199.00 / U S \$ 367.00$

For special $\mathrm{IOM}^{3}$ member rates please email

subscriptions@maney.co.uk

\section{For further information or to subscribe online please visit www.maney.co.uk}



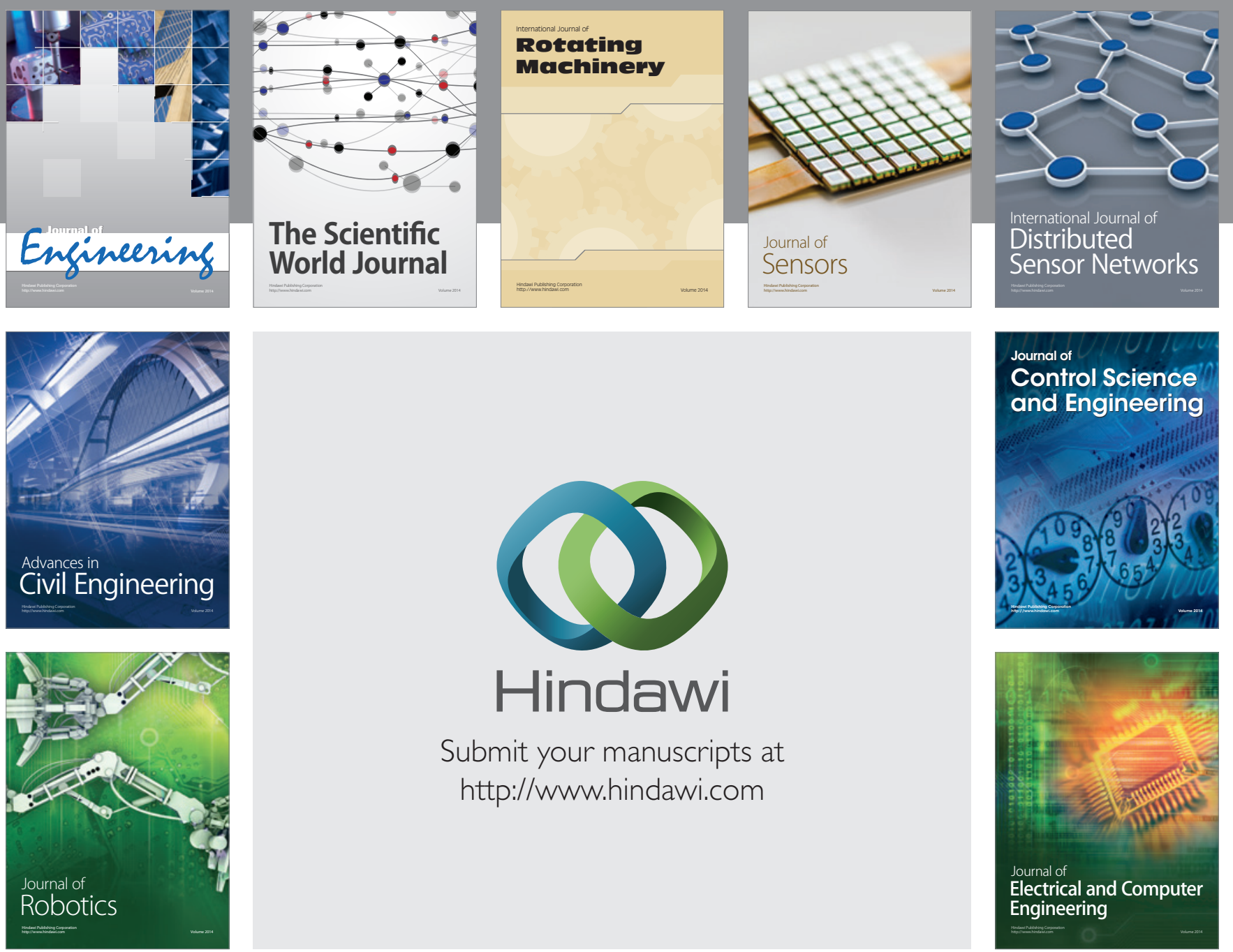

Submit your manuscripts at

http://www.hindawi.com
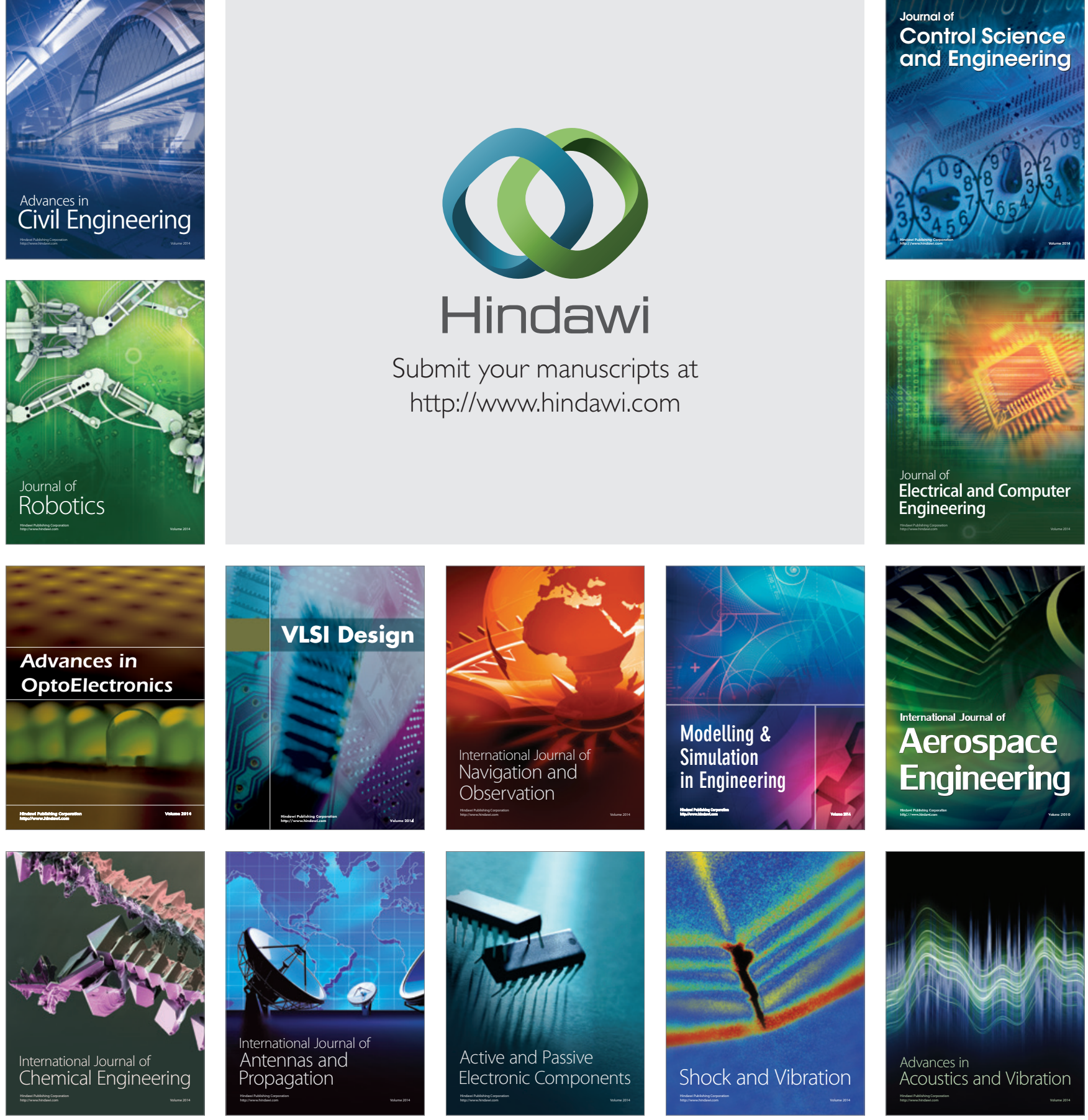